PAPER

Alternative constitutive relation for momentum transport of extended Navier-Stokes equations

To cite this article: Guo-Feng Han et al 2020 Chinese Phys. B 29124701

View the article online for updates and enhancements. 


\title{
Alternative constitutive relation for momentum transport of extended Navier-Stokes equations ${ }^{*}$
}

\author{
Guo-Feng Han(韩国锋) ${ }^{1}$, Xiao-Li Liu(刘晓丽) $)^{2, \dagger}$, Jin Huang(黄进) ${ }^{2}$, Kumar Nawnit ${ }^{2}$, and Liang Sun(孙亮) ${ }^{3}$ \\ ${ }^{1}$ Institute of Mechanics, Chinese Academy of Sciences, Beijing 100190, China \\ ${ }^{2}$ State Key Laboratory of Hydroscience and Engineering, Tsinghua University, Beijing 100084, China \\ ${ }^{3}$ PetroChina Research Institute of Petroleum Exploration \& Development, Beijing 100083, China
}

(Received 1 May 2020; revised manuscript received 28 June 2020; accepted manuscript online 1 September 2020)

The classical Navier-Stokes equation (NSE) is the fundamental partial differential equation that describes the flow of fluids, but in certain cases, like high local density and temperature gradient, it is inconsistent with the experimental results. Some extended Navier-Stokes equations with diffusion terms taken into consideration have been proposed. However, a consensus conclusion on the specific expression of the additional diffusion term has not been reached in the academic circle. The models adopt the form of the generalized Newtonian constitutive relation by substituting the convection velocity with a new term, or by using some analogy. In this study, a new constitutive relation for momentum transport and a momentum balance equation are obtained based on the molecular kinetic theory. The new constitutive relation preserves the symmetry of the deviation stress, and the momentum balance equation satisfies Galilean invariance. The results show that for Poiseuille flow in a circular micro-tube, self-diffusion in micro-flow needs considering even if the local density gradient is very low.

Keywords: extended Navier-Stokes equation, constitutive relation, momentum transport, mass diffusion

PACS: 47.10.ad, 47.10.ab, 91.65.My

DOI: $10.1088 / 1674-1056 / a b b 3 e 5$

\section{Introduction}

In the past decades, the applicability of the classical Navier-Stokes equation (NSE) of fluid dynamics has been questioned by some researchers. It is believed that there is an additional mass diffusion flux when the local density and temperature are very high. The mass diffusion is mass transport caused by molecular number density gradient or temperature gradient. A few so-called extended NSEs in which the fluid diffusion is taken into consideration, have been proposed. ${ }^{[1-8]}$ Brenner was the first to propose a bi-velocity model, which a volume velocity and a mass velocity are incorporated into. ${ }^{[1-4]}$ The difference between the two velocities is due to molecular diffusion. He deemed that in the Newtonian constitutive relation the volume velocity instead of the mass velocity should be used. A physical mechanism was not offered for this constitutive relation. Dadzie et al. ${ }^{[9-11]}$ and Abramov ${ }^{[12]}$ derived a Boltzmann equation with a diffusion term taken into account, based on statistical mechanics. However, the same assumption as Brenner's was adopted that the general Newtonian constitutive relation was still valid using an appropriate velocity. Chakraborty and Durst ${ }^{[8]}$ derived a new constitutive relation for fluid flow from the viewpoint of molecular transport. However, many analogies were assumed in the derivation, and the physical mechanism was not explained. Sambasivam ${ }^{[13]}$ attempted to derive the constitutive relation solely from the viewpoint of molecular motion. His formulation suggested that the change of the superficial area of the control volume can induce deviation stress, which has the same viscosity as the deviation stress induced by the strain rate. The contributions of the convection and diffusion termare in the same form. Therefore, the derivation of the relation between the volumetric strain rate and the deviation stress is inadequate.

The formulations ${ }^{[13]}$ of the constitutive relation for momentum transport and momentum balance equation are revised in this study, and a new constitutive relation and a momentum balance equation are presented from the molecular motion point of view. This study provides a foundation for extended NSEs. The new relation retains the symmetry of the deviation stress, and the proposed momentum balance equations satisfy Galilean invariance. Different models previously available from the literature are also compared and discussed.

\section{Derivation of new constitutive relation}

By the same method as that given by Sambasivam, ${ }^{[13]}$ the time-average momentum flux per unit time in direction $i$ through a micro-plane $\delta A$ parallel with the coordinate plane $j$ can be represented as follows (Fig. 1):

$$
\overline{\rho C_{i} C_{j}}=\overline{\rho\left(u_{i}+u_{i}^{\mathrm{M}}\right)\left(u_{j}+u_{j}^{\mathrm{M}}\right)}
$$

* Project supported by the National Natural Science Foundation of China-Outstanding Youth Foundation (Grant No. 51522903), the National Natural Science Foundation of China (Grant Nos. 11602276 and 51479094), and the Fund from the Key Laboratory for Mechanics in Fluid Solid Coupling Systems of the Chinese Academy of Sciences. 


$$
\begin{aligned}
& =\overline{\rho\left(u_{i} u_{j}+u_{i}^{\mathrm{M}} u_{j}+u_{i} u_{j}^{\mathrm{M}}+u_{i}^{\mathrm{M}} u_{j}^{\mathrm{M}}\right)} \\
& =\rho u_{i} u_{j}+\overline{\rho u_{i}^{\mathrm{M}} u_{j}}+\overline{\rho u_{i} u_{j}^{\mathrm{M}}}+\overline{\rho u_{i}^{\mathrm{M}} u_{j}^{\mathrm{M}}},
\end{aligned}
$$

where $\boldsymbol{C}$ is the molecular velocity, $\boldsymbol{u}$ is the macroscopic convection velocity, $\boldsymbol{u}^{\mathrm{M}}$ is the molecular thermal velocity, $\rho$ is the density, subscripts $i$ and $j$ indicate the coordinate directions, and the bar over variable denotes the time-averaged value. The first term on the right-hand side in Eq. (1) represents the momentum flux per area per time induced by convection velocity. The second and third term are momentum transports due to molecular thermal motion. When $i \neq j$, the fourth term equals zero, as molecular motions are independent of direction and isotropy. However, when $i=j$, this term results in the pressure caused by the time-averaged molecular motion. ${ }^{[13]}$

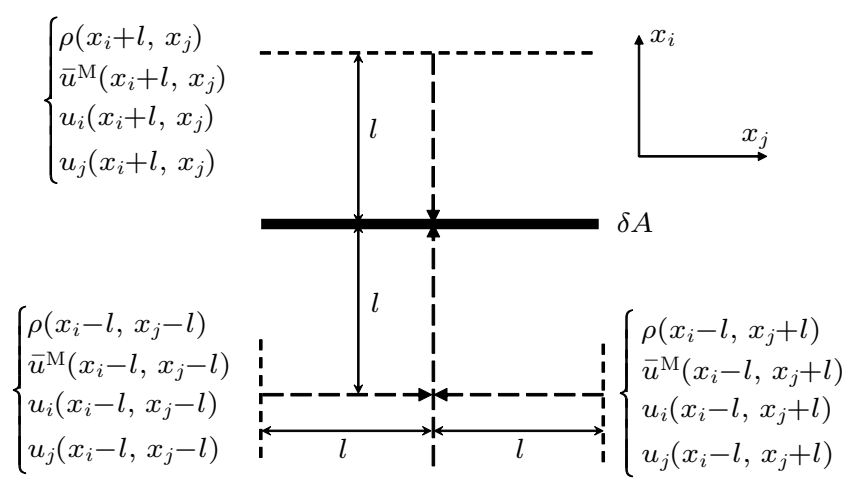

Fig. 1. $j$-direction momentum through $i$-direction micro-plane.

The second term of Eq. (1) indicates the $j$-direction momentum through the micro-plane $\delta A$ due to time-average molecular thermal motion in the $i$-direction. Because the molecular thermal motion is isotropic, the molecular thermal motion mean speed $\bar{u}^{\mathrm{M}}$ meets the equality $\overline{u^{\mathrm{M}}}=$ $\overline{\left|u_{i}^{\mathrm{M}}\right|}=\overline{\left|u_{j}^{\mathrm{M}}\right|}$. The average distance between the microplane $\delta A$ and the molecules passing through it from the positive and negative side is the mean free path $l$ of the molecules. Since the thermal motion of molecules is isotropic, the number of molecules moving in each direction is $1 / 6$. The momentum fluxes per unit area per time through the micro-plane $\delta A$ are $\frac{1}{6} \rho\left(x_{i}-l\right) \bar{u}^{\mathrm{M}}\left(x_{i}-l\right) u_{j}\left(x_{i}-l\right)$ and $-\frac{1}{6} \rho\left(x_{i}+l\right) \bar{u}^{\mathrm{M}}\left(x_{i}+l\right) u_{j}\left(x_{i}+l\right)$ from the $i$ and $-i$ directions respectively. Neglecting the quadratic terms of the partial derivative, the net momentum flux can be expressed as

$$
\begin{aligned}
& \frac{1}{6} \rho\left(x_{i}-l\right) \bar{u}^{\mathrm{M}}\left(x_{i}-l\right) u_{j}\left(x_{i}-l\right) \\
& -\frac{1}{6} \rho\left(x_{i}+l\right) \bar{u}^{\mathrm{M}}\left(x_{i}+l\right) u_{j}\left(x_{i}+l\right) \\
\approx & -\underbrace{\frac{1}{3} l \rho \bar{u}^{\mathrm{M}}}_{\mu}\left[\frac{1}{\rho} \frac{\partial \rho}{\partial x_{i}} u_{j}+\frac{1}{\overline{u^{\mathrm{M}}}} \frac{\partial \bar{u}^{\mathrm{M}}}{\partial x_{i}} u_{j}+\frac{\partial u_{j}}{\partial x_{i}}\right],
\end{aligned}
$$

where $\mu$ is the viscosity. The net momentum flux through unit area of the micro-plane per unit time, induced by molecular self-diffusion in the $i$-direction, can be expressed as ${ }^{[13]}$

$j_{i}=-\mu\left(\frac{1}{\rho} \frac{\partial \rho}{\partial x_{i}}+\frac{1}{\bar{u}^{\mathrm{M}}} \frac{\partial \bar{u}^{\mathrm{M}}}{\partial x_{i}}\right)=-\mu\left(\frac{1}{\rho} \frac{\partial \rho}{\partial x_{i}}+\frac{1}{2 T} \frac{\partial T}{\partial x_{i}}\right)$.

Self-diffusion is the spatial transport of mass, momentum, or energy caused by the number density or temperature gradient of the same kind of molecules. The second equal sign in the above equation is due to the assumption that the molecular velocity distribution satisfies the Maxwell distribution. Therefore, the molecular mean speed and the temperature are related by

$$
\bar{u}^{\mathrm{M}}=\sqrt{\frac{8 k T}{\pi M_{m}}},
$$

where $k$ is the Boltzmann constant, $M_{\mathrm{m}}$ is the molecular mass, and $T$ is the thermodynamic temperature. Therefore, the momentum transport due to the second term is given as

$$
j_{i} u_{j}-\mu \frac{\partial u_{j}}{\partial x_{i}} .
$$

The second term in the momentum transport expression (5) belongs to the deviation stress, and the first term is not a deviation stress because it does not satisfy Galilean invariance.

For the third term of Eq. (1), our explanation is much different from others'. ${ }^{\left[{ }^{8,13]}\right.}$ Due to the gradient of velocity $u_{i}$ in the $j$-direction, the number of molecules with thermal motion $\bar{u}^{\mathrm{M}}$ in the $+j$ direction and that in the $-j$ direction, moving through the micro-plane $\delta A$ per unit time interval by velocity $u_{i}$, are not equal. This means that the $j$-direction momentum is transported in the $i$-direction by velocity $u_{i}$ (Fig. 1). Therefore, the third term of momentum transport in Eq. (1) can be written as

$$
\begin{aligned}
& \frac{1}{6} \rho u_{i}\left(x_{j}-l\right) \bar{u}^{\mathrm{M}}-\frac{1}{6} \rho u_{i}\left(x_{j}+l\right) \bar{u}^{\mathrm{M}} \\
\approx & -\underbrace{\frac{1}{3} l \rho \bar{u}^{\mathrm{M}}}_{\mu} \frac{\partial u_{i}}{\partial x_{j}}=-\mu \frac{\partial u_{i}}{\partial x_{j}} .
\end{aligned}
$$

After an average time $\tau$, the molecular group, which is the fluid mass that can define a macroscopic quantity in a local equilibrium, passes through the micro-plane $\delta A$. The molecular group has a local density before time $\tau$, which is different from the instantaneous local density $\rho$. The density of the molecular group at that moment can be expressed as

$$
\left(\rho+\frac{\partial j_{k}}{\partial x_{k}} \tau\right)\left(1+\frac{\partial u_{k}}{\partial x_{k}} \tau\right)
$$

The Einstein summation convention is used in this equation. Therefore, the fourth term in Eq. (1) can be expressed as

$$
\begin{aligned}
\overline{\rho u_{i}^{\mathrm{M}} u_{j}^{\mathrm{M}}} & =\left(\rho+\frac{\partial j_{k}}{\partial x_{k}} \tau\right)\left(1+\frac{\partial u_{k}}{\partial x_{k}} \tau\right) \overline{u_{i}^{\mathrm{M}} u_{i}^{\mathrm{M}}} \\
& \approx \frac{1}{3}\left(1+\frac{1}{\rho} \frac{\partial j_{k}}{\partial x_{k}} \tau+\frac{\partial u_{k}}{\partial x_{k}} \tau\right) \overline{\rho\left(u^{\mathrm{M}}\right)^{2}} .
\end{aligned}
$$


The average molecular motion time $\tau$ can be identified with the time needed to travel the distance of the molecular mean free path $l$ at the mean speed $\bar{u}^{\mathrm{M}}$. Subsequently $\tau$ can be expressed as

$$
\tau=\frac{\alpha l}{\bar{u}^{\mathrm{M}}}
$$

where $\alpha$ is a coefficient, which is equal to 1 in the present case. According to a more rigorous theory, ${ }^{[14]}$ the average distance between the last collision and the micro-plane $\delta A$ is $(2 / 3) l$, which implies that $\alpha=2 / 3$.

The gas pressure obeys the relation

$$
p=\frac{1}{3} \rho \overline{\left(u^{\mathrm{M}}\right)^{2}}
$$

Therefore, the fourth term in Eq. (1) can be written as

$$
\begin{aligned}
\overline{\rho u_{i}^{\mathrm{M}} u_{j}^{\mathrm{M}}} & =p+\alpha \mu\left(\frac{1}{\rho} \frac{\partial j_{k}}{\partial x_{k}}+\frac{\partial u_{k}}{\partial x_{k}}\right) \\
& =p+\lambda\left(\frac{1}{\rho} \frac{\partial j_{k}}{\partial x_{k}}+\frac{\partial u_{k}}{\partial x_{k}}\right)
\end{aligned}
$$

where $\lambda$ is a viscosity parameter. The first term determines the pressure, and the second and third term correspond to the deviation stress. They are consistent with Galilean invariance. In fluid mechanics, the second viscosity coefficient is defined as

$$
\mu^{\prime}=\frac{2}{3} \mu-\lambda
$$

According to a more rigorous molecular kinetic theory, $\alpha=$ $2 / 3,{ }^{[14]}$ at this time $\mu^{\prime}=0$. This is consistent with the usual assumptions of fluid mechanics.

The time variation of density is ignored in the derivation of the momentum transport induced by the first and second term in Eq. (1). In those terms, the time partial derivative of density is always multiplied by the space partial derivatives of other variables, thus can be neglected. Combining the aforementioned momentum transport, the momentum balance equation can be written as follows:

$$
\frac{\partial\left(\rho u_{j}\right)}{\partial t}+\frac{\partial\left(\rho u_{i} u_{j}\right)}{\partial x_{i}}=-\frac{\partial p}{\partial x_{j}}-\frac{\partial \tau_{i j}}{\partial x_{i}}-\frac{\partial\left(j_{i} u_{j}\right)}{\partial x_{i}}+\rho g_{j}
$$

In Eq. (13), the integrated constitutive relation for the deviation stress is

$$
\tau_{i j}=-\mu\left(\frac{\partial u_{j}}{\partial x_{i}}+\frac{\partial u_{i}}{\partial x_{j}}\right)+\lambda \delta_{i j}\left(\frac{1}{\rho} \frac{\partial j_{k}}{\partial x_{k}}+\frac{\partial u_{k}}{\partial x_{k}}\right) .
$$

If the self-diffusion-induced terms are neglected, equation (13) is the momentum balance equation of the classical NSEs, and equation (14) has the form of a classical general Newtonian constitutive relation.

\section{Model comparisons and discussion}

The constitutive relation in Eq. (14) shows that the deviation stress is caused by the velocity gradient and molecular self-diffusion. In this constitutive relation there is more deviation stress term due to molecular self-diffusion than in the classical generalized Newtonian constitutive relation. In the momentum balance equation, each molecular self-diffusion term has a corresponding velocity gradient term, but their forms are different.

There are some differences between the constitutive relations developed in this study and in other theories developed for momentum transport. The equation provided by Brenner replaces the mass velocity in the classical generalized Newtonian constitutive relation with a volume velocity, which leads to an additional diffusion term. The proposed momentum balance equation is very similar to the result of Sambasivam, which is presented as follows: ${ }^{[13]}$

$$
\begin{aligned}
\tau_{i j}^{\mathrm{T}}= & -\mu\left(\frac{\partial u_{j}}{\partial x_{i}}+\frac{\partial u_{i}}{\partial x_{j}}\right)+\frac{2}{3} \mu \delta_{i j} \frac{\partial u_{k}}{\partial x_{k}} \\
& +j_{i} u_{j}+j_{j} u_{i}-\frac{2}{3} \delta_{i j} j_{k} u_{k} .
\end{aligned}
$$

But the new momentum balance equation has one less momentum transport term related to mass diffusion. Other major difference is in the sign and the form of the terms related to the second viscosity. The two mechanisms causing momentum transport have different effects on the molecular groups. The diffusion just reduces the fluid density, while the convection velocity gradient causes volume to expand. However, they are assumed to work in the same way as that in Sambasivam's work. ${ }^{[13]}$ Reddy et al. ${ }^{[15]}$ proposed a re-casted Navier-Stokes equations based on the generalized Newtonian constitutive relation as follows:

$$
\tau_{v}^{\mathrm{RNS}}=\tau_{v}^{\mathrm{NS}}+\frac{k_{m}^{2}}{\rho} \nabla \rho \nabla \rho-k_{m} \boldsymbol{U}_{v} \nabla \rho-k_{m} \nabla \rho \boldsymbol{U}_{v},
$$

where $k_{\mathrm{m}}$ is the molecular diffusion coefficient, $\tau_{v}^{\mathrm{NS}}$ is the deviation stress and converted from the generalized Newton constitutive equation by substituting $\boldsymbol{U}_{v}$ into it, and it is equal to the deviation stress of the generalized Newton constitutive. The average volume velocity $\boldsymbol{U}_{v}$ has the following relation with the conventional convection velocity $\boldsymbol{u}$ :

$$
\boldsymbol{u}=\boldsymbol{U}_{v}-\frac{k_{m}}{\rho} \nabla \rho
$$

This constitutive relation is consistent with all thermomechanical properties. The momentum balance equation has two additional terms similar to those proposed in this paper, but other terms are different.

New extended NSEs can be composed of the proposed momentum balance equation and momentum constitutive relation in this study, the continuity equation and energy equation given by Sambasivam. ${ }^{[13]}$ It is easy to verify that these 
equations satisfy Galilean invariance, which is proved in the Appendix, because the energy equation is added to only by dissipation terms related to mass diffusion compared with the classical NSEs. For closed systems, there is no mass diffusion at the system boundaries. Considering that NSEs obey the second law of thermodynamics, the equations of the extended NSEs also conform to this law.

The momentum equation with additional diffusion terms can be obtained directly from a Boltzmann equation involving a diffusion term in the literature, ${ }^{[10]}$ but the classical generalized Newtonian constitutive relation has also been considered for the constitutive relation of deviation stress. Dadzie $^{[16]}$ pointed out that these relations cannot ensure conservation of local angular momentum. Similarly, in the case of Abramov, ${ }^{[12]}$ the momentum equation is not conservative. In the present study the deviation stress is symmetrical. According to the momentum balance equation proposed in this study, ignoring the gravity term, the following local angular momentum balance equation exists: ${ }^{[17]}$

$$
\frac{\partial l_{i}}{\partial t}=-\frac{\partial}{\partial x_{l}}\left[u_{l} l_{i}+\varepsilon_{i j k} r_{j}\left(j_{l} u_{k}+p \delta_{k l}+\tau_{k l}\right)\right]-\varepsilon_{i j k} j_{j} u_{k}
$$

The last term on the right-hand side of Eq. (18) is the angular momentum transport caused by mass diffusion. Therefore, the momentum balance equation proposed in this study does not satisfy the conservation of local angular momentum. But for the closed system, the total angular momentum is conserved. Furthermore, the conservation of local angular momentum is not necessary. The local conservation of angular momentum requires that the vector sum of the external moment vectors acting on a particle system should be zero in magnitude, which is composed of fixed particles. Equation (18) is only applicable for the macroscopic system, and the molecules that make up of this system are not completely fixed due to molecule diffusion. This is the origin of the angular momentum transport in Eq. (18).

The constitutive relation for momentum transport in this study is based on the primary theory of molecular motion theory. A more rigorous derivation should use statistical mechanics to obtain a modified Boltzmann equation to take into account the molecular self-diffusion term. ${ }^{[18]}$ No specific form of the self-diffusion term has yet been widely accepted. ${ }^{[9-12]}$ Based on a modified Boltzmann equation, a constitutive relation for momentum transport and energy transport can be derived in the same way as that used by Enskog. ${ }^{[19]}$

For a circular micro-tube, the mass flux of convection for isothermal Poiseuille flow can be written as

$$
M^{\mathrm{C}}=-\frac{\pi \rho R^{4}}{8 \mu} \frac{\partial p}{\partial x_{1}},
$$

where $R$ is the radius of the circular tube, and $x_{1}$ is the coordinate along the length of the circular tube. Assuming the ideal gas state equation to be applicable, the mass flux of molecular self-diffusion can be directly obtained from Eq. (3) as follows:

$$
\begin{aligned}
M^{\mathrm{D}} & =j_{1} \cdot \pi R^{2}=-\frac{\mu \pi R^{2}}{\rho} \frac{\partial \rho}{\partial x_{1}}=-\frac{\mu \pi R^{2}}{\frac{p M_{m}}{R_{m} T}} \frac{\partial}{\partial x_{1}}\left(\frac{p M_{m}}{R_{m} T}\right) \\
& =-\frac{\mu \pi R^{2}}{p} \frac{\partial p}{\partial x_{1}}
\end{aligned}
$$

where $R_{\mathrm{m}}$ is the universal gas constant. The total mass flux is composed of convection mass flux and diffusion mass flux. This result is similar to that given by Stamatiou et al. ${ }^{[20]}$ but with different coefficients. Dividing Eq. (20) by Eq. (19), the mass flux ratio can be described by the following formula:

$$
\mathrm{RDC}=\frac{M^{\mathrm{D}}}{M^{\mathrm{C}}}=\frac{8 \mu^{2}}{p \rho R^{2}} .
$$

The ratios between convection flux to molecular selfdiffusion flux of helium under different pressures and tube radii can be obtained by using Eq. (21), and the results are shown in Fig. 2. The thermo-physical properties are cited from the NIST Chemistry Webbook. It can be found that the lower the pressure, the higher the contribution of the molecular selfdiffusion is. When the pressure is below $1 \mathrm{MPa}$ and the radius of the circular tube is $100 \mathrm{~nm}$, the mass flux of molecular selfdiffusion exceeds $19 \%$ that of convection. If the circular tube radius is less than $10 \mathrm{~nm}$, the molecular self-diffusion flux cannot be ignored even when the pressure reaches up to $10 \mathrm{MPa}$. For a 10-nm-radius pipe, when the pressure is less than $1 \mathrm{MPa}$, the flux of molecular self-diffusion is more than 10 times that of convection. It is notable that in these calculation results the density gradient is not taken into consideration. Therefore, for a micro-scale flow, the molecular self-diffusion is a very important mechanism of the total mass transport, and should be considered even when the local density gradient is very small.

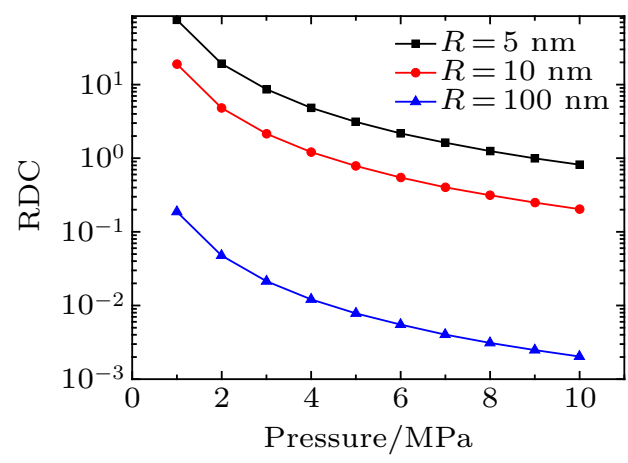

Fig. 2. Plots of mass flux ratio of self-diffusion to convection for circular micro-tubes of different radii.

When studying the problems of high density and temperature gradients such as shock wave structures, some researchers believe that mass diffusion must be considered. ${ }^{[13,21]}$ The analysis in this case shows that for micro-flow, mass diffusion must also be considered. This point has also been proved by other 
studies through using the Navier-Stokes equation modified by the mass diffusion term to fit the experimental results of high Knudsen number micro-flow. ${ }^{[13,22,23]}$ Although these support this research, they cannot strictly demonstrate the correctness of the model proposed in this study, which requires a lot of subsequent experimental researches.

\section{Conclusions and perspectives}

In this study, a new constitutive relation for fluid momentum transport and a momentum balance equation are derived from the viewpoint of microscopic molecular motion. In contrast to the assumptions of classical NSEs, the momentum transport caused by the molecular self-diffusion is also considered. The proposed momentum balance equation is consistent with Galilean invariance. The deviation stress tensor is symmetric in this constitutive relation. Unlike phenomenological methods or analogies in the literature, the method proposed in this study is based on the molecular motion theory, and can offer a physical foundation for the extended Navier-Stokes equations. This study is just a preliminary theory based on molecular motion, and a more rigorous derivation would require a modified Boltzmann equation to consider the diffusion term. Through the analysis of flows in micro-scale circular tubes, it is found that for a micro-scale flow, the molecular selfdiffusion should still be taken into consideration even when the local density gradient is very low.

\section{Data availability statement}

The data that support the findings of this study are available from the corresponding author upon reasonable request.

\section{Appendix A: Proof of Galilean invariance}

Assuming that the coordinate system $x^{\prime} y^{\prime} z^{\prime}$ moves at a uniform velocity $\boldsymbol{v}_{0}$ relative to the coordinate system $x y z$, the Galilean transformation is as follows:

$$
\left\{\begin{array}{l}
\boldsymbol{r}^{\prime}=\boldsymbol{r}-\boldsymbol{v}_{0} t \\
t^{\prime}=t,
\end{array}\right.
$$

where $\boldsymbol{r}^{\prime}, t^{\prime}, \boldsymbol{r}$, and $t$ are the radius vector and time in the coordinate system $x^{\prime} y^{\prime} z^{\prime}$ and $x y z$, respectively. The following can be obtained from Eq. (A1):

$$
\boldsymbol{u}^{\prime}=\boldsymbol{u}-\boldsymbol{v}_{0},
$$

where $\boldsymbol{u}^{\prime}$ and $\boldsymbol{u}$ are the velocity in the coordinate system $x^{\prime} y^{\prime} z^{\prime}$ and $x y z$, respectively. For any function $A(\boldsymbol{r}, t)$, according to the chain rule, the following equations hold:

$$
\begin{aligned}
\frac{\partial A}{\partial t^{\prime}} & =\frac{\partial t}{\partial t^{\prime}} \cdot \frac{\partial A}{\partial t}+\frac{\partial \boldsymbol{r}}{\partial t^{\prime}} \cdot \frac{\partial A}{\partial \boldsymbol{r}} \\
& =\frac{\partial A}{\partial t}+\frac{\partial t}{\partial t^{\prime}} \cdot \boldsymbol{v}_{0} \cdot \frac{\partial A}{\partial \boldsymbol{r}}=\frac{\partial A}{\partial t}+\boldsymbol{v}_{0} \cdot \frac{\partial A}{\partial \boldsymbol{r}}
\end{aligned}
$$

$$
\frac{\partial A}{\partial \boldsymbol{r}^{\prime}}=\frac{\partial A}{\partial t} \cdot \frac{\partial t}{\partial \boldsymbol{r}^{\prime}}+\frac{\partial A}{\partial \boldsymbol{r}} \cdot \frac{\partial \boldsymbol{r}}{\partial \boldsymbol{r}^{\prime}}=\frac{\partial A}{\partial \boldsymbol{r}}
$$

that is,

$$
\frac{\partial}{\partial t^{\prime}}=\frac{\partial}{\partial t}+v_{0} \cdot \nabla, \quad \nabla^{\prime}=\nabla
$$

For convenience, the continuity equation proposed by Sambasivam ${ }^{[13]}$ and the momentum equation and momentum constitutive relation proposed in this study are written in the form of the following vectors:

$$
\begin{aligned}
& \frac{\partial \rho}{\partial t}+\nabla \cdot(\rho \boldsymbol{u})=-\nabla \cdot \boldsymbol{j} \\
& \frac{\partial}{\partial t}(\rho \boldsymbol{u})+\nabla \cdot(\rho \boldsymbol{u u})=-\nabla \cdot(p \boldsymbol{I})-\nabla \cdot \boldsymbol{\tau}-\nabla \cdot(\boldsymbol{j u})+\rho \boldsymbol{g} \\
& \boldsymbol{\tau}=-\mu\left[\nabla \boldsymbol{u}+(\nabla \boldsymbol{u})^{\mathrm{T}}\right]+\lambda \boldsymbol{I}\left(\frac{1}{\rho} \nabla \cdot \boldsymbol{j}+\nabla \cdot \boldsymbol{u}\right) \\
& \boldsymbol{j}=-\mu\left(\frac{1}{\rho} \nabla \rho+\frac{1}{2 T} \nabla T\right)
\end{aligned}
$$

where $\boldsymbol{I}$ is the second-order unit tensor. Combining Eq. (A5), considering that none of the viscosity, density and temperature changes with the coordinate system, the following formula obviously holds:

$$
j=-\mu\left(\frac{1}{\rho} \nabla^{\prime} \rho+\frac{1}{2 T} \nabla^{\prime} T\right)=j^{\prime} .
$$

Therefore, the mass diffusion flux $\boldsymbol{j}$ is objective. According to Eqs. (A2), (A5), and (A10), the following equation holds for the deviation stress $\tau$ :

$$
\begin{aligned}
\boldsymbol{\tau}= & -\mu\left[\nabla \boldsymbol{u}+(\nabla \boldsymbol{u})^{\mathrm{T}}\right]+\lambda \boldsymbol{I}\left(\frac{1}{\rho} \nabla \cdot \boldsymbol{j}+\nabla \cdot \boldsymbol{u}\right) \\
= & -\mu\left\{\nabla^{\prime}\left(\boldsymbol{u}^{\prime}+\boldsymbol{v}_{0}\right)+\left[\nabla\left(\boldsymbol{u}^{\prime}+\boldsymbol{v}_{0}\right)\right]^{\mathrm{T}}\right\} \\
& +\lambda \boldsymbol{I}\left[\frac{1}{\rho} \nabla^{\prime} \cdot \boldsymbol{j}^{\prime}+\nabla^{\prime} \cdot\left(\boldsymbol{u}^{\prime}+\boldsymbol{v}_{0}\right)\right] \\
= & -\mu\left[\nabla^{\prime} \boldsymbol{u}^{\prime}+\left(\nabla^{\prime} \boldsymbol{u}\right)^{\mathrm{T}}\right]+\lambda \boldsymbol{I}\left(\frac{1}{\rho} \nabla^{\prime} \cdot \boldsymbol{j}^{\prime}+\nabla^{\prime} \cdot \boldsymbol{u}^{\prime}\right)=\boldsymbol{\tau}^{\prime} .
\end{aligned}
$$

Therefore, the deviation stress $\tau$ is also objective, and has Galilean invariance. From Eqs. (A2), (A5), continuity equation (A6) and Eq. (A10), the following equation can be obtained:

$$
\begin{aligned}
& \frac{\partial \rho}{\partial t^{\prime}}+\nabla^{\prime} \cdot\left(\rho \boldsymbol{u}^{\prime}\right) \\
= & \frac{\partial \rho}{\partial t}+\boldsymbol{v}_{0} \cdot \nabla \rho+\nabla \cdot\left[\rho\left(\boldsymbol{u}-\boldsymbol{v}_{0}\right)\right] \\
= & \frac{\partial \rho}{\partial t}+\boldsymbol{v}_{0} \cdot \nabla \rho-\nabla \cdot\left(\rho \boldsymbol{v}_{0}\right)+\nabla \cdot(\rho \boldsymbol{u}) \\
= & \frac{\partial \rho}{\partial t}+\nabla \cdot(\rho \boldsymbol{u})=-\nabla \cdot \boldsymbol{j}=-\nabla^{\prime} \cdot \boldsymbol{j}^{\prime} .
\end{aligned}
$$

Therefore, the continuity equation (A6) has Galilean invariance. For the momentum transport equation, the following equations can also be obtained:

$$
\frac{\partial}{\partial t^{\prime}}\left(\rho \boldsymbol{u}^{\prime}\right)=\frac{\partial}{\partial t}\left[\rho\left(\boldsymbol{u}-\boldsymbol{v}_{0}\right)\right]+\boldsymbol{v}_{0} \cdot \nabla\left[\rho\left(\boldsymbol{u}-\boldsymbol{v}_{0}\right)\right]
$$




$$
\begin{aligned}
= & \frac{\partial}{\partial t}(\rho \boldsymbol{u})-\frac{\partial}{\partial t}\left(\rho \boldsymbol{v}_{0}\right) \\
& +\boldsymbol{v}_{0} \cdot \nabla(\rho \boldsymbol{u})-\boldsymbol{v}_{0} \cdot \nabla\left(\rho \boldsymbol{v}_{0}\right), \\
\nabla^{\prime} \cdot\left(\rho \boldsymbol{u}^{\prime} \boldsymbol{u}^{\prime}\right)= & \nabla \cdot\left[\boldsymbol{\rho}\left(\boldsymbol{u}-\boldsymbol{v}_{0}\right)\left(\boldsymbol{u}-\boldsymbol{v}_{0}\right)\right] \\
= & \nabla \cdot(\rho \boldsymbol{u} \boldsymbol{u})-\nabla \cdot\left(\rho \boldsymbol{u} \boldsymbol{v}_{0}\right) \\
& -\nabla \cdot\left(\rho \boldsymbol{v}_{0} \boldsymbol{u}\right)+\nabla \cdot\left(\rho \boldsymbol{v}_{0} \boldsymbol{v}_{0}\right), \\
\nabla^{\prime} \cdot\left(\boldsymbol{j}^{\prime} \boldsymbol{u}^{\prime}\right)= & \nabla \cdot\left[\boldsymbol{j}\left(\boldsymbol{u}-\boldsymbol{v}_{0}\right)\right]=\nabla \cdot(\boldsymbol{j u})-\nabla \cdot\left(\boldsymbol{j} \boldsymbol{v}_{0}\right), \\
\nabla^{\prime} \cdot \boldsymbol{\tau}^{\prime}= & \nabla \cdot \boldsymbol{\tau} .
\end{aligned}
$$

Considering that in the case of the Galilean transformation (A1), neither of the pressure $p$ and the acceleration of gravity $\boldsymbol{g}$ changes with the coordinate system, so the following holds:

$$
\nabla \cdot(p \boldsymbol{I})=\nabla^{\prime} \cdot\left(p^{\prime} \boldsymbol{I}\right), \boldsymbol{g}=\boldsymbol{g}^{\prime} .
$$

The following equation can be obtained from Eqs. (A13)(A16):

$$
\begin{aligned}
& \frac{\partial}{\partial t^{\prime}}\left(\rho \boldsymbol{u}^{\prime}\right)+\nabla^{\prime} \cdot\left(\rho \boldsymbol{u}^{\prime} \boldsymbol{u}^{\prime}\right) \\
= & \frac{\partial}{\partial t}(\rho \boldsymbol{u})+\nabla \cdot(\rho \boldsymbol{u} \boldsymbol{u})-\frac{\partial}{\partial t}\left(\rho \boldsymbol{v}_{0}\right)-\nabla \cdot\left(\rho \boldsymbol{u} \boldsymbol{v}_{0}\right) .
\end{aligned}
$$

The following can be obtained from Eq. (A6):

$$
\frac{\partial}{\partial t}\left(\rho \boldsymbol{v}_{0}\right)+\nabla \cdot\left(\rho \boldsymbol{u} \boldsymbol{v}_{0}\right)=-\nabla \cdot\left(\boldsymbol{j} \boldsymbol{v}_{0}\right) .
$$

Substituting Eqs. (A19) and (A7) into the right side of Eq. (A18), the following equation can be obtained:

$$
\begin{aligned}
& \frac{\partial}{\partial t^{\prime}}\left(\rho \boldsymbol{u}^{\prime}\right)+\nabla^{\prime} \cdot\left(\rho \boldsymbol{u}^{\prime} \boldsymbol{u}^{\prime}\right) \\
= & -\nabla \cdot(p \boldsymbol{I})-\nabla \cdot \boldsymbol{\tau}-\nabla \cdot(\boldsymbol{j} \boldsymbol{u})+\nabla \cdot\left(\boldsymbol{j} \boldsymbol{v}_{0}\right)+\rho \boldsymbol{g} .
\end{aligned}
$$

From Eqs. (A15)-(A17), equation (A20) can be written in the following form:

$$
\frac{\partial}{\partial t^{\prime}}\left(\rho \boldsymbol{u}^{\prime}\right)+\nabla^{\prime} \cdot\left(\rho \boldsymbol{u}^{\prime} \boldsymbol{u}^{\prime}\right)
$$

$$
=-\nabla^{\prime} \cdot\left(p^{\prime} \boldsymbol{I}\right)-\nabla^{\prime} \cdot \boldsymbol{\tau}^{\prime}-\nabla^{\prime} \cdot\left(\boldsymbol{j}^{\prime} \boldsymbol{u}^{\prime}\right)+\rho \boldsymbol{g}^{\prime} .
$$

This shows that the momentum balance equation has Galilean invariance.

In summary, the momentum balance equation, momentum constitutive relation, and continuity equation, proposed in this work, all have their own Galilean invariance.

\section{References}

[1] Brenner H 2005 Physica A 34911

[2] Brenner H 2005 Physica A 34960

[3] Brenner H 2006 Physica A $\mathbf{3 7 0} 190$

[4] Brenner H 2009 Physica A 3883391

[5] Brenner H 2010 Physica A 3894026

[6] Brenner H 2012 Int. J. Eng. Sci. 5467

[7] Oettinger H C 2005 Beyond Equilibrium Thermodynamics (New Jersey: Wiley and Sons) pp. 51-63

[8] Chakraborty S and Durst F 2007 Phys. Fluids 19088104

[9] Dadzie S K, Reese J M and McInnes C R 2008 Physica A 3876079

[10] Dadzie S K and Reese J M 2012 Phys. Lett. A 376967

[11] Dadzie S K 2012 Phys. Lett. A 3763223

[12] Abramov R V 2017 Physica A 484532

[13] Sambasivam R 2013 Extended Navier-Stokes equations: derivations and applications to fluid flow problems, $\mathrm{Ph}$. D. Dissertation (Erlangen: University of Erlangen) pp. 18-38

[14] Kennard E H 1938 Kinetic theory of gases: with an introduction to statistical mechanics (New York and London: McGraw-Hill Book Company) p. 140

[15] Reddy M H L, Dadzie S K, Ocone R, Borg M K and Reese J M 2019 J. Phys. Commun. 3105009

[16] Dadzie S K and Reese J M 2012 Phys. Rev. E 85041202

[17] ttinger H C, Struchtrup H and Liu M 2009 Phys. Rev. E 80056303

[18] Han G F, Liu X L, Huang J, Nawnit K and Sun L 2020 Phys. Fluids 32 022001

[19] Chapman S and Cowling T G 1970 The mathematical theory of nonuniform gases, 3rd edn. (Cambridge: Cambridge University Press) pp. 110-131

[20] Stamatiou A, Dadzie S K and Reddy M H L 2019 J. Phys. Commun. 3 125012

[21] Greenshields C J and Reese J M 2007 J. Fluid Mech. 580407

[22] Lv Q, Liu X, Wang E and Wang S 2013 Phys. Rev. E 88013007

[23] Lv Q, Wang E, Liu X and Wang S 2014 Microfluid Nanofluid 16841 